Commun. Korean Math. Soc. 27 (2012), No. 3, pp. 477-482

http://dx.doi.org/10.4134/CKMS.2012.27.3.477

\title{
ON PARTITION CONGRUENCES FOR OVERCUBIC PARTITION PAIRS
}

\author{
BYUNGCHAN KIM
}

\begin{abstract}
In this note, we investigate partition congruences for a certain type of partition function, which is named as the overcubic partition pairs in light of the literature. Let $\overline{c p}(n)$ be the number of overcubic partition pairs. Then we will prove the following congruences:
\end{abstract}

$$
\overline{c p}(8 n+7) \equiv 0 \quad(\bmod 64) \quad \text { and } \quad \overline{c p}(9 m+3) \equiv 0 \quad(\bmod 3) .
$$

\section{Introduction}

In a paper [3], H.-C. Chan initiated the study of the cubic partitions by showing a close relation between a certain type of partition function and Ramanujan's cubic continued fraction. Cubic partition function $c(n)$ is defined by

$$
\sum_{n=0}^{\infty} c(n) q^{n}=\frac{1}{(q ; q)_{\infty}\left(q^{2} ; q^{2}\right)_{\infty}} .
$$

Here and in the sequel, we will use the following standard $q$-series notation:

$$
(a ; q)_{\infty}:=\prod_{n=1}^{\infty}\left(1-a q^{n-1}\right),|q|<1 .
$$

Motivated by his works $([3,4,5])$, many partition congruences for analogous partition functions have been investigated. In particular, the author studied its overpartition analog [7] in which the overcubic partition function $\bar{c}(n)$ was defined by

$$
\sum_{n=0}^{\infty} \bar{c}(n) q^{n}=\frac{(-q ; q)_{\infty}\left(-q^{2} ; q^{2}\right)_{\infty}}{(q ; q)_{\infty}\left(q^{2} ; q^{2}\right)_{\infty}}
$$

Received April 26, 2011.

2010 Mathematics Subject Classification. 11P83.

Key words and phrases. partition, cubic partition, overcubic partition pair. 
More recently, H. Zhao and Z. Zhong [13] investigated congruences for the following partition function:

$$
\sum_{n=0}^{\infty} c p(n) q^{n}=\frac{1}{(q ; q)_{\infty}^{2}\left(q^{2} ; q^{2}\right)_{\infty}^{2}} .
$$

Since $c p(n)$ counts a pair of cubic partitions, we will call $c p(n)$ the number of cubic partition pairs. We can interpret $c p(n)$ as the number of 4-color partitions of $n$ with colors $r, y, o$, and $b$ subject to the restriction that the colors $o$ and $b$ appear only in even parts.

In this article, we will investigate congruence properties of the following partition function $\overline{c p}(n)$, which is named as the number of overcubic partition pairs in light of (1.1) and (1.2),

$$
\sum_{n=0}^{\infty} \overline{c p}(n) q^{n}=\frac{(-q ; q)_{\infty}^{2}\left(-q^{2} ; q^{2}\right)_{\infty}^{2}}{(q ; q)_{\infty}^{2}\left(q^{2} ; q^{2}\right)_{\infty}^{2}}
$$

We can interpret $\overline{c p}(n)$ as the number of 4-color partitions of $n$ with colors $r$, $y, o$, and $b$ subject to the restriction that the colors $o$ and $b$ appear only in even parts in which we may overline the first occurrence parts.

First, we will investigate congruences for $\overline{c p}(n)$ modulo 64 by using arithmetic properties of quadratic forms.

Theorem 1. For all nonnegative integers $n$,

$$
\overline{c p}(8 n+7) \equiv 0 \quad(\bmod 64) .
$$
forms.

Next, we find the following congruence for $\overline{c p}(n)$ via the aid of modular

Theorem 2. For all nonnegative integers $n$,

$$
\overline{c p}(9 n+3) \equiv 0 \quad(\bmod 3) .
$$

Combining the above two congruences, we can obtain the following congruences.

Corollary 3. For all nonnegative integer $n$,

$$
\overline{c p}(72 n+39) \equiv 0 \quad(\bmod 192) .
$$

This paper is organized as follows. In Section 2, we review a 2-adic generating function of the overpartition function and basic properties of modular forms. In Section 3, we prove our results.

\section{Preliminaries}

Let $\bar{P}(q)$ be

$$
\bar{P}(q)=\frac{(-q ; q)_{\infty}}{(q ; q)_{\infty}}
$$


and $\varphi(q)$ be

$$
\varphi(q)=\sum_{n=1}^{\infty} q^{n^{2}} .
$$

Then, the coefficients of $\varphi(q)^{k}=\sum_{n \geq 1} c_{k}(n) q^{n}$ are the number of representations of $n=n_{1}^{2}+\cdots+n_{k}^{2}$ where each $n_{i}$ is a positive integer. From Mahlburg's paper [10], we know that

$$
\bar{P}(q)=1+\sum_{k=1}^{\infty}(-2)^{k} \varphi(-q)^{k} .
$$

By reducing (2.1) modulo 64, we arrive at

$$
\begin{aligned}
\overline{C P}(q)= & \sum_{n=0}^{\infty} \overline{c p}(n) q^{n} \\
= & \bar{P}(q)^{2} \bar{P}\left(q^{2}\right)^{2} \\
= & \left(1-2 \varphi(-q)+2^{2} \varphi(-q)^{2}-\cdots\right)^{2}\left(1-2 \varphi\left(-q^{2}\right)+2^{2} \varphi\left(-q^{2}\right)^{2}-\cdots\right)^{2} \\
= & 1-4 \varphi(-q)-4 \varphi\left(-q^{2}\right)+12 \varphi(-q)^{2}+12 \varphi\left(-q^{2}\right)^{2}+16 \varphi(-q) \varphi\left(-q^{2}\right) \\
& +16 \varphi(-q)^{4}+16 \varphi\left(-q^{2}\right)^{4}-48 \varphi(-q)^{2} \varphi\left(-q^{2}\right)-48 \varphi(-q) \varphi\left(-q^{2}\right)^{2} \\
& -48 \varphi(-q)^{2} \varphi\left(-q^{2}\right)^{2}+144 \varphi(-q)^{2} \varphi\left(-q^{2}\right)^{2}-32 \varphi(-q)^{3} \\
& -32 \varphi\left(-q^{2}\right)^{3}(\bmod 64) .
\end{aligned}
$$

Now we give some basic properties of modular functions. For more details on this subject, consult [12]. Define

$$
\Gamma=S L_{2}(\mathbb{Z}), \Gamma_{0}(N):=\left\{\left(\begin{array}{ll}
a & b \\
c & d
\end{array}\right) \in \Gamma: c \equiv 0 \quad(\bmod N)\right\} .
$$

For a meromorphic function $f$ on the complex upper half plane $\mathcal{H}$, define the slash operator by

$$
f \mid\left(\begin{array}{ll}
a & b \\
c & d
\end{array}\right):=f\left(\frac{a z+b}{c z+d}\right) .
$$

We say a meromorphic function $f$ is a modular function on $\Gamma_{0}(N)$ if $f$ is invariant under the slash operator. Let $\mathcal{M}_{0}\left(\Gamma_{0}(N)\right)$ denote the vector space of modular functions on $\Gamma_{0}(N)$. For a prime $p$, we define the $U_{p}$-operator as follows. If $f(q)$ has a Fourier expansion $f(q)=\sum a(n) q^{n}$, then

$$
U_{p} f(z):=\sum a(p n) q^{n} .
$$

It is well known that $U_{p} f(z) \in \mathcal{M}_{0}\left(\Gamma_{0}(N p)\right)$ provided $f(z) \in \mathcal{M}_{0}\left(\Gamma_{0}\left(N p^{2}\right)\right)$. Let $\eta(z)=q^{1 / 24}(q ; q)_{\infty}$ be Dedekind eta function, where $q=\exp (2 \pi i z)$ and $z$ is in the upper half complex plane $\mathcal{H}$. For a fixed $N$ and integers $r_{i}$ 's, a 
function of the form

$$
f(z):=\prod_{\substack{n \mid N \\ n>0}} \eta(n z)^{r_{n}}
$$

is called an $\eta$-quotient. By the famous theorem of Newman [11], we can determine when an $\eta$-quotient becomes a modular function. We call the orbits of $\mathbb{Q} \cup\{i \infty\}\left(\bmod \Gamma_{0}(N)\right)$ the cusps of $\Gamma_{0}(N)$. Moreover, by the work of Ligozat [9], we can calculate the order of the $\eta$-quotient $f$ at the cusps $c / d$ of $\Gamma_{0}(N)$ provided $f \in \mathcal{M}_{0}\left(\Gamma_{0}(N)\right)$. Recall that if $p \mid N$ and $f \in \mathcal{M}_{0}\left(\Gamma_{0}(p N)\right)$, then $U_{p} f \in \mathcal{M}_{0}\left(\Gamma_{0}(N)\right)$. The following theorem gives bounds on the order of $U_{p} f$ at cusps of $\Gamma_{0}(N)$ in terms of the order of $f$ at cusps of $\Gamma_{0}(p N)$.

Theorem 4 (Theorem 4 of [6]). Let $p$ be a prime and $\pi(n)$ be the highest power of $p$ dividing $n$. Suppose that $f \in \mathcal{M}_{0}\left(\Gamma_{0}(p N)\right)$, where $p \mid N$ and $\alpha=c / d$ is a cusp of $\Gamma_{0}(N)$. Then,

$$
\operatorname{ord}_{\alpha} U_{p} f \geq \begin{cases}\frac{1}{p} \operatorname{ord}_{\alpha / p} f, & \text { if } \pi(d) \geq \frac{1}{2} \pi(N), \\ \operatorname{ord}_{\alpha / p} f, & \text { if } 0<\pi(d)<\frac{1}{2} \pi(N), \\ \min _{0 \leq \beta \leq p-1} \operatorname{ord}_{(\alpha+\beta) / p} f, & \text { if } \pi(d)=0 .\end{cases}
$$

\section{Proofs of theorems}

We now start the proof of Theorem 1.

Proof of Theorem 1. For a quadratic form $Q=\sum_{i=1}^{k} a_{i} x_{i}^{2}$, let $R(n, Q)$ be the number of representations of $n$ by $n=a_{1} n_{1}^{2}+a_{2} n_{2}^{2}+\cdots+a_{k} n_{k}^{2}$, where $a_{i}$ 's and $n_{i}$ 's are positive integers. By (2.2), it suffices to show the following congruences:

$$
\begin{aligned}
R\left(8 n+7, x_{1}^{2}\right) & \equiv R\left(8 n+7,2 x_{1}^{2}\right) \\
& \equiv R\left(8 n+7, x_{1}^{2}+x_{2}^{2}\right) \\
& \equiv R\left(8 n+7,2 x_{1}^{2}+2 x_{2}^{2}\right) \equiv 0 \quad(\bmod 16), \\
R\left(8 n+7, x_{1}^{2}+2 x_{2}^{2}\right) & \equiv R\left(8 n+7, x_{1}^{2}+x_{2}^{2}+2 x_{3}^{2}\right) \\
& \equiv R\left(8 n+7, x_{1}^{2}+2 x_{2}^{2}+2 x_{3}^{2}\right) \\
& \equiv R\left(8 n+7, x_{1}^{2}+x_{2}^{2}+x_{3}^{2}+x_{4}^{2}\right) \\
& \equiv R\left(8 n+7,2 x_{1}^{2}+2 x_{2}^{2}+2 x_{3}^{2}+2 x_{4}^{2}\right) \\
& \equiv R\left(8 n+7, x_{1}^{2}+x_{2}^{2}+2 x_{3}^{2}+2 x_{4}^{2}\right) \equiv 0 \quad(\bmod 4), \\
R\left(8 n+7, x_{1}^{2}+x_{2}^{2}+x_{3}^{2}\right) & \equiv R\left(8 n+7,2 x_{1}^{2}+2 x_{2}^{2}+2 x_{3}^{2}\right) \equiv 0 \quad(\bmod 2) .
\end{aligned}
$$

Note that $x^{2} \equiv 0,1$, or $4(\bmod 8)$. By using this, we can see that there is no integer solution for $8 n+7=x_{1}^{2}+x_{2}^{2}+2 x_{3}^{2}$ and we also see that if $8 n+7=x_{1}^{2}+x_{2}^{2}+x_{3}^{2}+x_{4}^{2}$, then at least one of $x_{i}$ is different from others. By using a similar argument, we can verify the above congruences hold. 
Remark. By proceeding as in [8], one can prove that $\{n \in \mathbb{N}: \overline{c p}(n) \equiv 0$ $\left.\left(\bmod 2^{k}\right)\right\}$ has the arithmetic density 1 for any fixed positive integer $k$. As we focus on explicit congruences in this article, we will omit the proof of this fact.

Now we turn to the proof of Theorem 2.

Proof of Theorem 2. Let define $G(z)$ as

$$
G(z)=\frac{\eta^{2}(4 z) \eta^{4}(9 z) \eta^{2}(18 z)}{\eta^{4}(z) \eta^{2}(2 z) \eta^{2}(36 z)}
$$

Then, by applying Newman's theorem, we see that $G(z) \in \mathcal{M}\left(\Gamma_{0}(36)\right)$. By employing $U_{3}$ operator, we see that $U_{3} G(z) \in \mathcal{M}\left(\Gamma_{0}(12)\right)$ and arrive at

$$
U_{3} G(z)=\left(\sum_{n=0}^{\infty} \overline{c p}(3 n) q^{n}\right) \frac{\left(q^{3} ; q^{3}\right)_{\infty}^{4}\left(q^{9} ; q^{9}\right)_{\infty}^{2}}{\left(q^{12} ; q^{12}\right)_{\infty}^{2}}
$$

By applying Theorem 4, we can find lower bounds for the orders of $U_{3} G(z)$ at the cusps as follows:

\begin{tabular}{|c||c|c|c|c|c|c|}
\hline $\mathrm{d}$ & 1 & 2 & 3 & 4 & 6 & 12 \\
\hline $\operatorname{ord}_{c / d} U_{3} G(z) \geq$ & -6 & -6 & 2 & -6 & 1 & 0 \\
\hline
\end{tabular}

After multiplying $\Delta(z)^{2}=\eta^{48}(z)$ to $U_{3} G(z)$, we observe that $U_{3} G(z) \Delta(z)^{2}$ is in $\mathcal{M}_{24}\left(\Gamma_{0}(12)\right)$ and $U_{3} G(z) \Delta(z)^{2}$ is a holomorphic modular form and so is $U_{3}\left(U_{3} G(z) \Delta(z)^{2}\right)$. Moreover,

$$
\begin{aligned}
U_{3}\left(U_{3} G(z) \Delta(z)^{2}\right) & \equiv U_{3}\left(U_{3} G(z) \eta^{16}(3 z)\right) \quad(\bmod 3) \\
& \equiv U_{3}\left(\sum_{n=0}^{\infty} \overline{c p}(3 n) q^{n+2}\right) \frac{(q ; q)_{\infty}^{20}\left(q^{3} ; q^{3}\right)_{\infty}^{2}}{\left(q^{4} ; q^{4}\right)_{\infty}^{2}} \quad(\bmod 3) \\
& \equiv\left(\sum_{n=1}^{\infty} \overline{c p}(9 n-6) q^{n}\right) \frac{(q ; q)_{\infty}^{20}\left(q^{3} ; q^{3}\right)_{\infty}^{2}}{\left(q^{4} ; q^{4}\right)_{\infty}^{2}}(\bmod 3)
\end{aligned}
$$

Since the dimension of holomorphic modular form of weight 24 with level 12 is 49 , by Sturm's theorem, we can verify that $\overline{c p}(9 n-6) \equiv 0(\bmod 3)$ by checking up to $n=50$.

Remark. There is no single eta-quotient in $\mathcal{M}_{0}\left(\Gamma_{0}(12)\right)$ which coincides with $U_{3} G(z)$.

\section{References}

[1] G. E. Andrews and F. G. Garvan, Dyson's crank of a partition, Bull. Amer. Math. Soc. (N.S.) 18 (1988), no. 2, 167-171.

[2] B. C. Berndt, Number Theory in the Spirit of Ramanujan, American Mathematical Society, Providence, RI, 2006.

[3] H.-C. Chan, Ramanujan's cubic continued fraction and an analog of his "most beautiful identity", Int. J. Number Theory 6 (2010), no. 3, 673-680.

[4] _ Ramanujan's cubic continued fraction and Ramanujan type congruences for a certain partition function, Int. J. Number Theory 6 (2010), no. 4, 819-834. 
[5] Distribution of a certain partition function modulo powers of primes, Acta Math. Sin. (Engl. Ser.) 27 (2011), 625-634.

[6] B. Gordon and K. Hughes, Ramanujan congruences for $q(n)$, Analytic number theory (Philadelphia, Pa., 1980), 333-359, Lecture Notes in Math., 899, Springer, Berlin-New York, 1981.

[7] B. Kim, The overcubic partition function mod 3, Proceedings of Ramanujan Rediscovered 2009: A Conference in Memory of K. Venkatachaliengar on the Centenary of His Birth, Lecture Note Series of the Ramanujan Mathematical Society 14 (2010), 157-163.

[8] - Overpartition pairs modulo powers of 2, Discrete Math. 311 (2011), no. 10-11, 835-840.

[9] G. Ligozat, Courbes modulaires de genre 1, Bull. Soc. Math. France, Mem. 43. Supplement au Bull. Soc. Math. France Tome 103, no. 3. Societe Mathematique de France, Paris, 1975. $80 \mathrm{pp}$

[10] K. Mahlburg, The overpartition function modulo small powers of 2, Discrete Math. 286 (2004), no. 3, 263-267.

[11] M. Newman, Construction and application of a class of modular functions II, Proc. London Math. Soc. (3) 9 (1959), 373-387.

[12] K. Ono, Web of Modularity: arithmetic of the coefficients of modular forms and q-series, CBMS Regional Conference Series in Mathematics, 102. Published for the Conference Board of the Mathematical Sciences, Washington, DC, by the American Mathematical Society, Providence, RI, 2004.

[13] H. Zhao and Z. Zhong, Ramanujan type congruences for a partition function, The Electronic Lournal of Combinatorics 18 (2011), P. 58.

School of Liberal Arts

Seoul National University of Science and Technology

SeOul 139-743, Korea

E-mail address: bkim4@seoultech.ac.kr 